\title{
Influência da anestesia geral e bloqueio peribulbar em trabeculectomias: comparação visual e pressórica
}

\author{
Peribulbar blockage and general anesthesia influency in \\ trabeculectomies: visual and pressure effects
}

Carlos Buhler ${ }^{1}$, Juliana Almodin², Flavia Almodin³, Tadeu Cvintal ${ }^{4}$

\section{Resumo}

Objetivo: Avaliar os resultados de trabeculectomias (TRECs) com anestesia geral e bloqueio peribulbar, visando definir de forma indireta a influência das técnicas anestésicas no resultado da cirurgia. Métodos: Foram analisados os prontuários de 25 pacientes com glaucoma não controlado submetidos à trabeculectomia (TREC), sendo 11 com anestesia geral e 14 com anestesia peribulbar. Foram incluídos somente pacientes pseudofácicos com glaucoma e sem outras patologias oculares como retinopatia diabética ou doença macular relacionada a idade. A acuidade visual (AV), pressão intraocultar (PIO), número de medicações utilizadas, além de dados demográficos foram coletados retrospectivamente com 7,30, 6 meses e 1 ano de pós-operatório. Resultados: Ambos os grupos foram semelhantes nos quesitos demográficos, onde não houve diferença estatisticamente significante entre idade, sexo e raça. Não foram encontradas diferenças significativas nas variáveis estudadas em todas as avaliações, com significância estatística $(\mathrm{p}<0,05)$. Conclusão: Não encontramos diferenças entre os resultados de TRECs com anestesia geral e com bloqueio peribulbar, porém mais estudos com um número maior de pacientes são necessários para comprovar este achado.

Descritores: Glaucoma; Trabeculectomia; Anestesia geral; Anestesia local; Pressão intraocular

\begin{abstract}
Objective: To evaluate trabeculetomies results with peribulbar anesthesia and general anesthesia and to watch the influence by this two techniques in the surgery result. Methods: It was analyzed charts of 25 patients with uncontrolled glaucoma that undergone trabeculectomy, 11 patients had general anesthesia and 14 with peribulbar anesthesia. It was included in this study pseudophakic patients with glaucoma. We excluded other ocular patologies such as diabetic retinopathy and macular degeneration. Visual acuity, intraocular pressure, number of medications and demographic data were collected. The data were analyzed in 7 days, 30 days, 6 months and 1 year after surgery. Results: Both groups were similar in demographic data, with no statically significance among age, sex and race. We didn't find significant differences in the variables studied in all evaluations, with statisctically significance $(\mathrm{p}<0.05)$. Conclusion: There was no statistically difference between patients submitted to surgery with general anesthesia or peribulbar anesthesia. We need a larger group of patients to comprove it.
\end{abstract}

Keywords: Glaucoma; Trabeculectomy; Anestesia, general; Anestesia, local; Intraocular pressure

\footnotetext{
${ }^{1}$ Instituto de Oftalmologia Tadeu Cvintal - São Paulo (SP), Brasil;

${ }^{2}$ Instituto de Oftalmologia Tadeu Cvintal - São Paulo (SP), Brasil;

${ }^{3}$ Instituto de Oftalmologia Tadeu Cvintal - São Paulo (SP), Brasil;

${ }^{4}$ Instituto de Oftalmologia Tadeu Cvintal - São Paulo (SP), Brasil
}

Os autores declaram inexistir conflitos de interesse

Recebido para publicação em 28/2/2011 - Aceito para publicação em 7/11/2011 


\section{INTRODUÇÃO}

$A$ trabeculectomia (TREC) é uma das modalidades de cirurgias filtrantes mais usadas no controle da pressão intraocular (PIO). Ela consiste em criar uma comunicação entre a câmara anterior (CA) do olho com o espaço subconjuntival, permitindo o escoamento do humor aquoso (HA) e diminuindo a PIO. Existem alguns tipos de anestesia para realização dessa técnica cirúrgica, sendo elas a anestesia geral, a local (peribulbar, retrobulbar e subtenoniana) e a tópica. Todas as técnicas anestésicas visam à realização de uma cirurgia com serenidade, proporcionando a analgesia do paciente, o conforto, sangramento mínimo e prevenção da hipertensão ocular (HO).(1)

A anestesia geral tem sido indicada em casos de cirurgias em crianças, pacientes com deficiência mental, emocionalmente instáveis ou olhos com alguma alteração anatômica, como alta miopia e bulftalmos. ${ }^{(2)} \mathrm{Com}$ a melhora das drogas utilizadas na indução e manutenção da anestesia geral a variação da PIO pode ser mais bem controlada. ${ }^{(3-6)}$ A técnica peribulbar comprovadamente causa variação da PIO pelo aumento do volume intraorbitário causado pela injeção o anestésico. ${ }^{(7-9)}$

O objetivo desse estudo é avaliar os resultados das TRECs realizadas com anestesia geral e com bloqueio peribulbar, visando definir de forma indireta a influência dessas técnicas anestésicas no sucesso dessa cirurgia.

\section{Métodos}

Foram selecionados e avaliados os prontuários de pacientes com glaucoma não controlado clinicamente com medicação tópica ocular que foram submetidos à TREC com pelo menos um ano de pós-operatório de um serviço de referência na cidade de São Paulo (Centro de Oftalmologia Tadeu Cvintal). Foram incluídos pacientes com diagnóstico de glaucoma de ângulo aberto (GPAA), já submetidos à facectomia e que não apresentassem alguma comorbidade ocular que afetasse AV. Foram excluídos os pacientes que apresentassem glaucoma neovascular, afácicos, glaucoma uveítico, glaucoma congênito, pacientes que apresentassem doenças retinianas que pudessem influenciar na $\mathrm{AV}$ como degeneração macular relacionada à idade e retinopatia diabética, pacientes que apresentassem qualquer grau de opacidade cristaliniana ou que tinham cristalino transparente e que pudessem evoluir com algum grau de catarata.

Os prontuários foram revisados e anotados os dados demográficos: idade, sexo, raça, olho operado. A AV, a PIO e o número de medicações antiglaucomatosas foram verificados com sete, trinta, seis meses, e um ano de pós-operatório. A AV foi medida em escala Snellen e transformada para escala LogMAR.Consideramos como sucesso cirúrgico pacientes que mantiveram com $\mathrm{PIO}<$ 21 mmhg com ou sem medicação antiglaucomatosa. No grupo da anestesia geral foram incluídos 11 pacientes (44\%), sendo 6 mulheres e 5 homens com uma média de idade de 75 anos (variação de 61 a 84 anos). No grupo do bloqueio peribulbar foram incluídos 14 pacientes $(56 \%)$, sendo 12 homens e 2 mulheres com uma média de idade de 74,5 anos (variação de 49 a 92 anos).

A associação entre grupo de anestesia e as variáveis categóricas foi avaliada pelo Teste do Qui-quadrado ou Teste exato de Fisher, de acordo com a indicação.

Foram aplicados modelos de Análise de Variância para medidas repetidas para comparar as medidas de $\mathrm{AV}$ e de PIO entre os grupos ao longo do seguimento.

Os grupos foram comparados quanto ao número de medicações utilizadas ao longo do seguimento pelo Teste de Mann-Whitney para amostras independentes, aplicado em cada momento de avaliação. Dentro de cada grupo, as avaliações foram comparadas pelo Teste de Friedman para dados relacionados.

Foi adotado o nível de significância de 0,05 (a = $5 \%$ ) e utilizado o programa estatístico SPSS versão 15.0 for Windows para todas as análises estatísticas.

\section{Resultados}

Eliminando os pacientes que não se encaixavam nos critérios de inclusão, foram selecionados vinte e cinco pacientes, sendo que onze (44\%) foram submetidos à TREC com anestesia geral e quatorze $(56 \%)$ com anestesia peribulbar. Quanto às variáveis demográficas, não foi encontrado diferença estatística entre os grupos, tanto na ida$\mathrm{de}$, sexo e raça, indicando semelhança entre os grupos ( $\mathrm{p}>$ 0,05 em todas as comparações), conforme indica tabela 1 .

Quanto ao fator AV não foi observado efeito estatisticamente significante da interação entre o fator acima nos dois grupos $(p=0,148)$, indicando que os grupos apresentaram comportamento semelhante ao longo do tempo de seguimento.

Foi encontrado efeito estatisticamente significante do fator AV durante o seguimento nos dois grupos $(\mathrm{p}=0,001)$, com as seguintes diferenças:

- A média de AV na avaliação pré foi significantemente maior do que as médias das avaliações de $7 \mathrm{~d}$ PO $(\mathrm{p}<0,001)$ e $1 \mathrm{~m}$ PO $(\mathrm{p}=0,012)$;

- A média de AV na avaliação de $7 \mathrm{~d}$ PO foi 
Tabela 1

Características da amostra de pacientes

\begin{tabular}{|c|c|c|c|c|}
\hline \multirow[b]{2}{*}{ Variáveis } & \multicolumn{2}{|c|}{ Grupo } & \multirow[b]{2}{*}{$\begin{array}{c}\text { Amostra total } \\
(\mathrm{n}=25)\end{array}$} & \multirow[b]{2}{*}{ Valor de $\mathrm{p}$} \\
\hline & $\begin{array}{l}\text { Anestesia geral } \\
\quad(\mathrm{n}=11)\end{array}$ & $\begin{array}{l}\text { Bloqueio peribulbar } \\
\qquad(\mathrm{n}=14)\end{array}$ & & \\
\hline \multicolumn{5}{|l|}{ Olho $-\mathrm{n}(\%)$} \\
\hline Direito & $7(63,6)$ & $9(64,3)$ & $16(64,0)$ & 1,000 \\
\hline Esquerdo & $4(36,4)$ & $5(35,7)$ & $9(36,0)$ & \\
\hline \multicolumn{5}{|l|}{ Sexo - n (\%) } \\
\hline Feminino & $6(54,5)$ & $2(14,3)$ & $8(32,0)$ & 0,081 \\
\hline Masculino & $5(45,5)$ & $12(85,7)$ & $17(68,0)$ & \\
\hline \multicolumn{5}{|l|}{ Idade (anos) } \\
\hline Média (dp) & $75,4(6,3)$ & $73,1(11,7)$ & $74,1(9,6)$ & 0,575 \\
\hline Mediana & 75,0 & 74,5 & 75,0 & \\
\hline Mínimo - máximo & $61-84$ & $49-92$ & $49-92$ & \\
\hline \multicolumn{5}{|l|}{ Raça - n (\%) } \\
\hline Branca & $8(72,7)$ & $8(57,1)$ & $16(64,0)$ & 0,457 \\
\hline Negra & $2(18,2)$ & $2(14,3)$ & $4(16,0)$ & \\
\hline Parda & $1(9,1)$ & $4(28,6)$ & $5(20,0)$ & \\
\hline
\end{tabular}

Tabela 2

Acuidade Visual (LogMAR) ao longo do seguimento, segundo o grupo de anestesia

\begin{tabular}{|c|c|c|c|c|c|}
\hline \multirow[t]{2}{*}{ Grupo } & \multicolumn{5}{|c|}{ Avaliação } \\
\hline & Pré & 7d PO & $1 \mathrm{~m}$ PO & 6m PO & 1a PO \\
\hline \multicolumn{6}{|c|}{ Anestesia geral $(n=11)$} \\
\hline Média (dp) & $0,67(0,56)$ & $1,21(0,63)$ & $0,90(0,54)$ & $0,78(0,59)$ & $0,72(0,61)$ \\
\hline Mediana & 0,40 & 1,00 & 0,80 & 0,50 & 0,40 \\
\hline Mínimo / máximo & $0,0-1,6$ & $0,4-2,3$ & $0,1-1,9$ & $0,1-1,9$ & $0,1-1,9$ \\
\hline \multicolumn{6}{|c|}{ Bloqueio peribulbar $(n=14)$} \\
\hline Média (dp) & $0,99(0,76)$ & $1,26(0,69)$ & $1,19(0,72)$ & $1,04(0,82)$ & $1,16(1,00)$ \\
\hline Mediana & 0,70 & 1,00 & 0,80 & 0,70 & 0,80 \\
\hline Mínimo / máximo & $0,1-2,5$ & $0,4-2,5$ & $0,2-2,5$ & $0,0-2,5$ & $0,0-3,0$ \\
\hline \multicolumn{6}{|l|}{ Amostra total $(n=25)$} \\
\hline Média (dp) & $0,85(0,69)$ & $1,24(0,65)$ & $1,06(0,65)$ & $0,92(0,72)$ & $0,97(0,87)$ \\
\hline Mediana & 0,60 & 1,00 & 0,80 & 0,60 & 0,60 \\
\hline Mínimo / máximo & $0,0-2,5$ & $0,4-2,5$ & $0,1-2,5$ & $0,0-2,5$ & $0,0-3,0$ \\
\hline Efeito de interação & $p=0,148$ & & & & \\
\hline Efeito de avaliação & $\mathrm{p}=0,001$ & & & & \\
\hline Efeito de grupo & $p=0,337$ & & & & \\
\hline
\end{tabular}

significantemente menor do que as médias das avaliações de $1 \mathrm{~m}$ PO $(\mathrm{p}=0,032), 6 \mathrm{~m}$ PO $(\mathrm{p}=0,003)$ e 1 a PO (p $=0,046)$.

As comparações entre as demais avaliações não mostraram diferenças estatisticamente significantes ( $p>0,05$ em todas as demais comparações), conforme mostra tabela 2.

Quanto a PIO, não foi observado efeito estatisticamente significante da interação entre os grupos $(\mathrm{p}=0,680)$, indicando que os grupos apresentaram com- portamento semelhante ao longo do tempo de seguimento.

Foi encontrado efeito estatisticamente significante do fator PIO durante o seguimento nos dois grupos ( $\mathrm{p}<0,001)$, com as seguintes diferenças:

- A média de PIO na avaliação Pré foi significantemente maior do que as médias das demais avaliações ( $\mathrm{p}<0,001$ em todas as comparações);

- A média de PIO na avaliação de $7 \mathrm{~d}$ PO foi significantemente menor do que as médias das demais 
Tabela 3

Pressão Intraocular ( $\mathrm{mmHg}$ ) ao longo do seguimento, segundo o grupo de anestesia

\begin{tabular}{lccccc}
\hline Grupo & \multicolumn{5}{c}{ Avaliação } \\
\cline { 2 - 6 } & Pré & $\mathbf{7 d ~ P O}$ & $\mathbf{1 m}$ PO & $\mathbf{6 m ~ P O}$ & 1a PO \\
\hline $\begin{array}{l}\text { Anestesia geral (n = 11) } \\
\quad \text { Média (dp) }\end{array}$ & $18,4(2,4)$ & $7,5(4,0)$ & $11,6(2,1)$ & $11,1(2,3)$ & $11,6(2,9)$ \\
$\quad$ Mediana & 18,0 & 8,0 & 12,0 & 11,0 & 11,0 \\
$\quad$ Mínimo / máximo & $15-23$ & $2-14$ & $7-15$ & $8-15$ & $8-18$ \\
Bloqueio peribulbar (n = 14) & & & & & \\
$\quad$ Média (dp) & $21,4(6,0)$ & $8,1(4,7)$ & $11,4(2,7)$ & $11,0(3,0)$ & $13,1(5,6)$ \\
$\quad$ Mediana & 20,0 & 9,0 & 12,0 & 10,0 & 12,5 \\
$\quad$ Mínimo / máximo & $16-38$ & $0-14$ & $7-16$ & $6-18$ & $5-26$ \\
Amostra total (n = 25) & & & & $11,0(2,7)$ & $12,4(4,6)$ \\
$\quad$ Média (dp) & $20,0(4,9)$ & $7,8(4,4)$ & $11,5(2,4)$ & 11,0 \\
$\quad$ Mediana & 19,0 & 8,0 & 12,0 & 10,0 & 12,0 \\
$\quad$ Mínimo / máximo & $15-38$ & $0-14$ & $7-16$ & $6-18$ & $5-26$ \\
$\quad$ Efeito de interação & $\mathrm{p}=0,680$ & & & & \\
Efeito de avaliação & $\mathrm{p}<0,001$ & & & & \\
Efeito de grupo & $\mathrm{p}=0,196$ & & & \\
\hline
\end{tabular}

avaliações $(\mathrm{p}=0,003$ comparado ao $1 \mathrm{~m}$ PO, $\mathrm{p}=0,045$ comparado ao 6m PO e p =0,015 comparado ao 1a PO).

As comparações entre as demais avaliações não mostraram diferenças estatisticamente significantes $(\mathrm{p}$ $>0,05$ em todas as demais comparações), conforme a tabela 3.

Quanto ao número de medicações antiglaucomatosas, não foi encontrado efeito estatisticamente significante desse fator entre os grupos ( $p>0,05$ em todas as avaliações).

No Grupo anestesia geral foi encontrado efeito estatisticamente significante do fator avaliação $(\mathrm{p}<$ 0,001), onde:

O número de medicações na avaliação pré foi significantemente maior do que das demais avaliações;

$\mathrm{O}$ número de medicações na avaliação $1^{\mathrm{a}} \mathrm{PO}$ foi significantemente maior do que das avaliações de $7 \mathrm{~d}$ PO e $1 \mathrm{~m}$ PO;

As comparações entre as demais avaliações não mostraram diferenças estatisticamente significantes ( $p>0,05$ em todas as demais comparações).

No Grupo bloqueio peribulbar foi encontrado efeito estatisticamente significante do fator avaliação ( $\mathrm{p}<0,001)$, onde:

O número de medicações na avaliação pré foi significantemente maior do que das demais avaliações;

As comparações entre as demais avaliações não mostraram diferenças estatisticamente significantes ( $p>0,05$ em todas as demais comparações).

\section{Discussão}

A anestesia local é o procedimento de escolha para cirurgias do glaucoma, porém em algumas situações de glaucoma com lesão avançada do nervo óptico, a anestesia geral ainda é indicada visando controlar o aumento da PIO após bloqueio anestésico e evitar possível lesão adicional glaucomatosa. ${ }^{(2)}$ Muitos estudos já mostraram a variação da PIO tanto com anestesia geral quanto com bloqueio peribulbar. Na anestesia geral alguns estudos mostraram maior eficácia no controle da PIO utilizando drogas como propofol e alfentanil, sendo a primeira, a droga utilizada em nosso estudo. ${ }^{(5-10)} \mathrm{Em}$ um estudo com bloqueio peribulbar, a PIO mostrou-se elevada e diminuiu o fluxo sanguíneo ocular pulsátil nos minutos iniciais, porém mostrou recuperação total após $10 \mathrm{~min}^{(11)}$. Em outro estudo os pesquisadores concluíram que o aumento da PIO após bloqueio peribulbar pode ser lesivo em olhos glaucomatosos. ${ }^{(7)}$ Apesar dessas evidências o resultado funcional nem sempre pode ser determinado o que leva a busca de comparação dos resultados dessas técnicas anestésicas.

Em nosso estudo, os grupos tiveram comportamento semelhante quanto à $\mathrm{AV}$ no período analisado, não encontrando diferenças significantes entre os grupos ( $\mathrm{P}>0,05)$. A AV avaliada no sétimo pós-operatório foi significativamente menor que nas demais avaliações, efeito possivelmente causado pelo estado pós-cirúrgico recente, visto que nas demais avaliações o estado visual 
inicial foi quase que totalmente recuperado. Quanto a $\mathrm{PIO}$, os grupos se mostraram semelhantes, mostrando comportamento semelhante durante o seguimento. Em ambos os grupos os resultados da mediana da PIO esteve dentro do classificado como sucesso cirúrgico $(\mathrm{PIO}<21 \mathrm{mmHg}$ com ou sem medicação antiglaucomatosa) como demonstrado em outros estudos. ${ }^{(11,12,13)}$

Em resumo, não encontramos diferenças significativas entres os resultados de TRECs realizadas com anestesia geral e bloqueio peribulbar, porém o número de pacientes analisados é pequeno, é necessário mais estudos com um número maior de pacientes para comprovar os efeitos mostrados.

\section{RefERÊNCIAS}

1. McGoldrick KE, Foldes PJ. General anesthesia for ophthalmic surgery. Ophthalmol Clin North Am. 2006;19(2):179-91.

2. Paletta Guedes RA, Paletta Guedes VM, Assis de Castro Paletta J, Pereira da Silva AC. [Anesthesia in glaucoma surgery]. J Fr Ophtalmol. 2009;32(3):221-5. Review.

3. Eltzschig HK, Darsow R, Schroeder TH, Hettesheimer H, Guggenberger $\mathrm{H}$. Effect of tracheal intubation or laryngeal mask airway insertion on intraocular pressure using balanced anesthesia with sevoflurane and remifentanil. J Clin Anesth. 2001;13(4):264-7.

4. Eti Z, Yayci A, Umuroglu T, Gögü ${ }^{\circ}$ FY, Bozkurt N. The effect of propofol and alfentanil on the increase in intraocular pressure due to succinylcholine and intubation. Eur J Ophthalmol. 2000;10(2):105-9.

5. Jantzen JP. [Anesthesia and intraocular pressure]. Anaesthesist. 1988;37(8):458-69. German.
6. Zimmerman AA, Funk KJ, Tidwell JL. Propofol and alfentanil prevent the increase in intraocular pressure caused by succinylcholine and endotracheal intubation during a rapid sequence induction of anesthesia. Anesth Analg. 1996;83(4):814-7.

7. O'Donoghue E, Batterbury M, Lavy T. Effect on intraocular pressure of local anaesthesia in eyes undergoing intraocular surgery. Br J Ophthalmol. 1994;78(8):605-7.

8. Bowman R, Liu C, Sarkies N. Intraocular pressure changes after peribulbar injections with and without ocular compression. Br J Ophthalmol. 1996;80(5):394-7.

9. Lanini PG, Simona FS. [Change in intraocular pressure after peribulbar and retrobulbar injection: practical sequelae]. Klin Monbl Augenheilkd. 1998;212(5):283-5. German.

10. Lauretti GR, Lauretti CR, Lauretti-Filho A. Propofol decreases ocular pressure in outpatients undergoing trabeculectomy. J Clin Anesth. 1997;9(4):289-92.

11. Watkins R, Beigi B, Yates M, Chang B, Linardos E. Intraocular pressure and pulsatile ocular blood flow after retrobulbar and peribulbar anaesthesia. Br J Ophthalmol. 2001;85(7):7968 .

12. Mosaed S, Dustin L, Minckler DS. Comparative outcomes between newer and older surgeries for glaucoma. Trans Am Ophthalmol Soc. 2009;107:127-33.

13. Fernández S, Pardiñas N, Laliena JL, Pablo L, Díaz S, Pérez S, Honrubia FM. [Long-term tensional results after trabeculectomy. A comparative study among types of glaucoma and previous medical treatment]. Arch Soc Esp Oftalmol. 2009;84(7):345-51. Spanish.

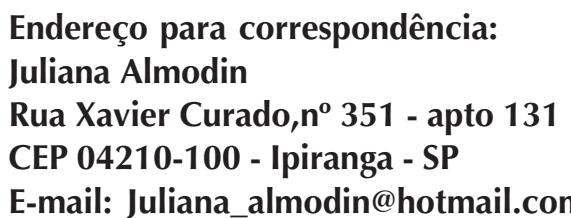

